\title{
Towards Applicable 3D User Interfaces for Everyday Working Environments
}

\author{
Frank Steinicke, Timo Ropinski, Gerd Bruder, and Klaus Hinrichs \\ Visualization and Computer Graphics (VisCG) Research Group \\ Institute of Computer Science, Westfälische Wilhelms-Universität Münster, \\ Einsteinstraße 62, 48149 Münster, Germany \\ \{fsteini, ropinski, g_brud01, khh\}@math.uni-muenster.de \\ http://viscg.uni-muenster.de
}

\begin{abstract}
Desktop environments represent a powerful user interface and have been used as the de facto standard human-computer interaction paradigm for over 20 years. But the rising demand of 3D applications dealing with complex datasets exceeds the capabilities of traditional interaction devices and two-dimensional displays. Such applications need more immersive and intuitive interfaces. In order to be accepted by the users, technology-driven solutions that require inconvenient instrumentation, e.g., stereo glasses or tracked gloves, should be avoided. Autostereoscopic display environments equipped with tracking systems enable humans to experience virtual 3D environments more naturally, for instance via gestures, without having to use annoying devices. However, currently these approaches are used only for specially designed or adapted applications. In this paper we introduce new 3D user interface concepts for such setups which require minimal instrumentation of the user and can be integrated easily in everyday working environments. We propose an interaction framework which supports simultaneous display of and simultaneous interaction with both monoscopic as well as stereoscopic contents. We identify the challenges for combined mouse-, keyboard- and gesture-based input paradigms in such an environment and introduce novel interaction strategies.
\end{abstract}

Keywords: HCI, autostereoscopic display environments, 3D user interfaces.

\section{Introduction}

In recent years 3D user interfaces (UIs) have become more and more popular and widespread due to the requirements of several application areas, where twodimensional desktop systems lack immersive and intuitive interaction. Bi-manual interactions or six degrees of freedom (DoFs) manipulations, which do not require much effort and are easy to learn even for non-experts, have increased the user's ability to perform complex interaction tasks. Current 3D UIs are technologydriven solutions providing more immersive exploration of and interaction with complex datasets, in particular by using stereoscopic projection and tracked six

C. Baranauskas et al. (Eds.): INTERACT 2007, LNCS 4662, Part I, pp. 546 559, 2007.

(C) IFIP International Federation for Information Processing 2007 
DoFs input devices. Although the costs for such a setup have reached a moderate level, common and even expert users hardly use these systems - even when $3 \mathrm{D}$ tasks have to be accomplished 3 . One reason for this is the inconvenient instrumentation required to allow immersive interactions, i.e., the user is forced to wear stereo glasses, tracked devices, gloves etc. 12]. Furthermore the most effective ways for humans to interact with synthetic $3 \mathrm{D}$ environments have not finally been resolved [36]. Devices that enable control over multiple DoFs simultaneously still involve problems, which are often avoided by the usage of their $2 \mathrm{D}$ counterparts - as a matter of fact $2 \mathrm{D}$ interactions are performed best with 2D devices 3917. However, while in real life humans are able to move and turn objects freely in a single motion, this natural interaction is absent in twodimensional interfaces; the user is forced to decompose 3D tasks into several 2D tasks. In addition, shortage of spatial input in typical 3D applications leads to the need to constantly switch modes. This procedure results in ineffectiveness, in particular when switching between manipulation and navigation techniques is required in a repetitive manner.

Most desktop-based 3D applications include three-dimensional content in combination with two-dimensional elements for graphical user interface (GUI) interaction. While 3D content usually benefits from stereoscopic display, 2D GUI items often do not require immersive visualization. For such a system current autostereoscopic (AS) displays can be used to view 3D data stereoscopically without wearing any devices [8]. Thus the user is able to perceive a stereoscopic image in a fixed area called sweet spot. When the AS display features, for instance, an optical head tracker, the user can even move in front of the display, while the tracking system can be further exploited to allow gesture-based interaction [11]. However, the separation of the stereo half images performed by an AS display (see Section 3.1) influences viewing of monoscopic content in such a way that essential elements of the GUI are distorted. Although some AS devices can display monoscopic content, simultaneous display of mono- as well as stereoscopic content is not supported. Thus, simultaneous viewing requires an additional conventional display to show the monoscopic content. But only few applications support rendering of a stereoscopic window on a different display. Nevertheless, problems arise from decoupling interaction and visualization; interactions with 2D GUI elements have to be performed on the 2D screen, whereas $3 \mathrm{D}$ content is displayed stereoscopically on an AS display.

In this paper we introduce new 3D user interface concepts as a solution to the lack of spatial input and intuitive interaction techniques for direct manipulation of mono- as well as stereoscopic content in desktop environments. We propose an AS display environment and present a framework which enables to display arbitrary shaped areas of the GUI either in a mono- or in a stereoscopic way. Furthermore, the framework allows interaction between both "worlds" and thus opens up new vistas for human-computer interaction (HCI). Hence, the user can interact with any 2D or 3D application via familiar mouse/keyboard devices in combination with natural gestures. 
The remainder of this paper is organized as follows. Section 2 summarizes related work. In Section 3 we describe the proposed setup, while Section 4 introduces interaction strategies for such everyday working environments. Section 5 presents implementation details. The results of an experimental evaluation are discussed in Section 6. Section 7 concludes the paper and gives an overview about future work.

\section{Related Work}

AS Display Environments. In 2000, the Heinrich-Hertz-Institute built an AS display system consisting of a gaze tracker, a head tracker and a hand tracker [1]. The head tracker gives the user a look-around capability, while the gaze tracking activates different applications on the desktop. The hand tracker enables the user to navigate and manipulate objects in $3 \mathrm{D}$ space via simple gestures, where computer vision is the major technological factor influencing the type of gesture that are supported. Similar approaches support gesture-based interactions by tracking the users hand and fingers with magnetic fields [23] or optical-based solutions 2. These approaches rather address tracking technologies than advanced $3 \mathrm{D}$ user interfaces. Although these systems potentially support novel forms of interaction, they are restricted to specific applications designed for these setups [2] simultaneous display of and simultaneous interaction with both monoscopic and stereoscopic content is not considered.

Simultaneous Monoscopic and Stereoscopic Display. Although current stereo-in-a-window systems [522] show stereoscopic content either in one window time-sequentially or using filtering techniques, these technologies are restricted to only one rectangular window and still require glasses. Hardware-based approaches have been proposed to display monoscopic and stereoscopic content simultaneously on one AS display [13. However, interaction concepts have not yet been developed for these displays, and these systems only exist as prototype solutions. Due to the lack of simultaneous display most interaction approaches only propose improvements for interactions either in 2D using monoscopic display or in 3D using stereoscopic display, but they do not combine both worlds. The interaction with stereoscopic content using two-dimensional strategies involves further problems, for instance, monoscopic representation of the mouse cursor disturbs stereoscopic perception and therefore precise interactions become more difficult to perform.

3D User Interfaces. In recent years, many frameworks have been proposed which extend 2D GUIs for operating systems (OSs) to so called 3D desktops, but also existing OSs evolve to 3D and include depth information [116]. These approaches provide a virtual 3D space in which 2D GUI elements are replaced by three-dimensional counterparts. Hence, more space is available to display further information. Although these environments provide a fancy visualization, it has not been investigated in how far they improve the interaction process, since they force the user to perform $3 \mathrm{D}$ interactions where $2 \mathrm{D}$ interactions are 
intended. Due to the mentioned shortcomings of virtual reality (VR) interfaces, hybrid approaches have been proposed which combine $2 \mathrm{D}$ and $3 \mathrm{D}$ interaction using different display or interaction technologies [421]. For example, Benko et al. have discussed techniques to grab monoscopically displayed objects from a projection screen in order to view them stereoscopically using a head mounted display [4. However, an instrumentation of the user is still required.

Bi-Manual Interactions. When interacting with the hands numerous factors have to be considered. With respect to the tasks, the hands need to be moved symmetrically or asymmetrically, some tasks can be performed better with the dominant, others with the non-dominant hand. Also the used input devices have a major impact on the way how bi-manual interactions are performed. For instance, the used devices can be alike (e.g., keyboard and keyboard) or different (e.g., mouse and keyboard), and they can support different DoFs or involve constraints.

These approaches are applied in everyday tasks as well as in most user interfaces. Writing on a sheet of paper, when one hand holds the pencil while the other fixes the sheet, involves asymmetrical interactions. In many computer games navigation tasks are performed by the dominant hand using the mouse, while status changes are accomplished with the non-dominant hand via keyboard shortcuts. Interaction techniques for large-screen displays or VR environments often involve symmetrical bi-manual manipulation in order to scale or rotate virtual objects.

However, the combination of traditional devices and gestures in AS display environments that run common 3D applications has not been considered until now. The aim of this paper is not to debate the validity of desktop-based interaction concepts - there is no need to throw away 40 years of 2D UI research or the benefits of technology-driven VR approaches. The objective is to explore in how far these concepts can mutually adapt to each other in order to provide efficient interfaces that will be accepted by users as setups for their daily working environments.

\section{Proposed System Setup}

In this section we present the setup which we believe has the potential to be accepted by the users since natural as well as immersive interactions are supported, whereas instrumentation of the user is avoided.

\subsection{Autostereoscopic Display Environment}

On current AS displays users can see 3D data without wearing any instruments, for example by using lenticular rasters [8]. The lenticular screen is a molded plastic sheet that forms dozens of tiny lenses per inch. This raster operates as a beam splitter and ensures that the pixels displayed in each odd column are seen with the left eye, while the pixels displayed in each even column are seen with 


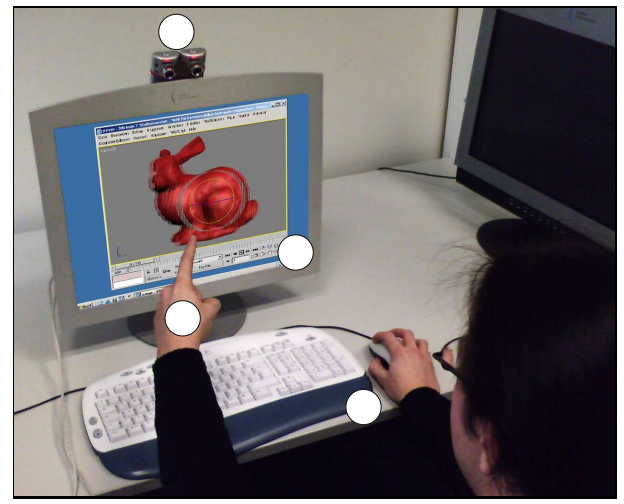

Fig. 1. 3D user interface setup includes (A) an AS display, (B) traditional mouse and keyboard, and (C) stereo-based camera setup. (D) The user applies gestures in order to perform 3D manipulations of a 3D scene. Due to the lenticular sheet the user perceives a stereoscopic image of the virtual scene displayed in 3D Studio Max.

the right eye. If the viewer positions her head in certain viewing positions, she sees a different image with each eye resulting in a stereo image. When leaving a sweet spot to a neighboring position, the stereo half images have to be swapped in order to maintain the stereoscopic effect.

The separation of the stereo half images influences viewing of monoscopic content in such a way that the most essential elements of the GUI are distorted. Therefore, we have implemented a software framework (see Section 5), which provides full control over the GUI of the OS. Thus, any region or object can be displayed either mono- or stereoscopically. Furthermore, we are able to catch the entire content of any 3D graphics application based on OpenGL or DirectX. Our framework allows to change the corresponding function calls in order to modify the visualization. The interaction performed in our setup is primarily based on mouse and keyboard (see Figure 11). However, we have extended these devices with more natural interfaces.

\subsection{Stereo-Based Trackingsystem}

AS displays can be equipped with eyes or head tracking systems to automatically adjust the two displayed images and the corresponding raster. Thus, the user perceives a stereo image in a larger region. Vision-based trackers enable non-intrusive, markerless computer vision based modules for HCI. When using computer vision techniques several features can be tracked, e.g., the eyes for head tracking, but it is also possible to track fingers in order to interpret simple as well as intuitive gestures in 3D. Pointing with the fingertip, for example, is an easy and natural way to select virtual objects.

As depicted in Figure1, we use a stereo-based camera setup consisting of two USB cameras each having a resolution of $640 \times 480$ pixels. They are attached to the top of the AS display in order to track the position and orientation of 
certain objects. Due to the known arrangement of the cameras, the pose of geometric objects, e.g., user's hands, can be reconstructed by 3D reprojection. Besides pointing actions, even some simple gestures signaling stop, start, left and right can be recognized. These gesture input events can be used to perform 3D manipulations, e.g., to rotate or translate virtual objects (see Figure 1).

\section{3D User Interface Concepts}

In the system setup described in the previous section, traditional input devices can be combined with gesture-based paradigms. There exist similar setups for artificial environments which are restricted to applications exclusively designed for or adapted to the specific environment [11. Hence, concepts developed for these restricted setups are not applicable in daily working environments for common applications. With the described framework we have full control over the GUI of the OS, in particular any arbitrarily shaped region can be displayed either monoor stereoscopically, and each 3D application can be modified appropriately. The implementation concepts are explained in Section 5. In the following subsections we discuss implications and introduce several universal interaction techniques that are usable for any 3D application.

\subsection{Universal Exploration}

As mentioned in Section 3.1 our framework enables us to control any content of an application based on OpenGL or DirectX. Virtual scenes in such applications are often defined by so-called display lists. Using our framework enables us to hijack and modify these lists. Among other possibilities this allows us to change the viewpoint in a virtual scene. Hence several navigation concepts can be realized that are usable for any $3 \mathrm{D}$ application.

Head Tracking. Since binocular vision is essential for depth perception, stereoscopic projections are mainly exploited to give a better insight into complex three-dimensional datasets. Although stereoscopic displays improve depth perception, viewing static images is limited, because other important depth cues, e.g, motion parallax phenomena, cannot be observed. Motion parallax denotes the fact that when objects or the viewer move, objects which are farther away from the viewer seem to move more slowly than objects closer to the viewer. To reproduce this effect, head tracking and view-dependent rendering is required. This can be achieved by exploiting the described tracking system (see Section 3.2). When the position and orientation of the user's head is tracked, this pose is mapped to the virtual camera defined in the $3 \mathrm{D}$ scene; furthermore the position of the lenticular sheet is adapted. Thus, the user is able to explore 3D datasets (to a certain degree) only by moving the tracked head. Such viewdependent rendering can also be integrated for any 3D application based on OpenGL. 

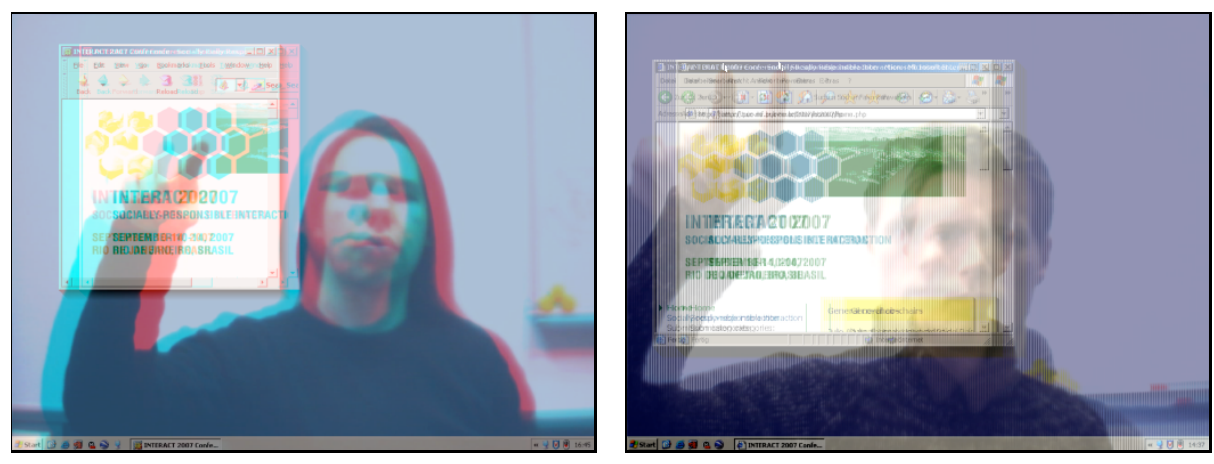

Fig. 2. Screenshot of an AS desktop overlaid with a transparent image of the user in (left) anaglyph mode and (right) vertical interlaced mode

Universal 3D Navigation and Manipulation Techniques. However, exploration only by head tracking is limited; object rotation is restricted by the tracking system, e.g. to $60^{\circ}$. Almost any interactive $3 \mathrm{D}$ application provides navigation techniques to explore virtual data from arbitrary viewpoints. Although many of these concepts are similar, e.g., mouse-based techniques to pan, zoom, rotate etc., 3D navigation as well as manipulation across different applications can become confusing due to various approaches.

The main idea to solve this shortcoming is to provide for different 3D applications the same universal paradigms to interact with a virtual scene. We use gestures to translate, scale or rotate objects or to move, fly or walk through a virtual environment. Moreover, individual strategies supported by each application can be used further on, e.g., by mouse- or keyboard-based interaction.

We have implemented these navigational concepts by using gestures based on virtual hand techniques [6]. Therefore, a one-to-one mapping in terms of translational and rotational mappings between the movements of the user's hand and the virtual scene is applied. Thus the user can start an arbitrary 3D application, activate gesture recognition and afterwards, the user can manipulate the scene by the combination of mouse, keyboard and gestures. Other concepts, such as virtual flying, walking etc. can be implemented, for instance, by virtual pointer approaches [6].

\subsection{Stereoscopic Facetop Interaction}

Besides depth information about the user's head and hand, we also exploit the images captured by the stereo-cameras mounted on top of the AS display (see Figure 10. Since the image planes of the cameras are coplanar and the distance between their lenses approximates the interpupillary distance of $\approx 65 \mathrm{~mm}$, both images compose a stereoscopic image of the user. Due to the full control over the GUI, we are able to display both half images transparently into the corresponding columns of the AS display - one image into the even columns, one into the odd ones. Hence, the user sees her image superimposed on the GUI as a transparent 
overlay; all desktop content can still be seen, but users appear to themselves as a semi-transparent image, as if looking through a window in which they can see their own reflection. This visualization can also be used in order to enable stereo-based face-to-face collaboration which is not topic of this paper.

The technique of superimposing the user's image on top of the display has been used recently in the Facetop system [20. More recently, Sony has released the Eyetoy that enables gesture interaction. In both approaches the user performs $2 \mathrm{D}$ interactions on the screen by $3 \mathrm{D}$ gestures, and visual feedback is given by displaying captured images of the user. However, besides gesturing for twodimensional control, e.g., moving the mouse cursor by pointing, a stereo-based camera setup allows to use multiple DoF to enable 3D interaction. Furthermore, we use the stereoscopic projection of the user. This provides not only visual feedback about the position of the cursor on the screen surface, but also about its depth in order to simplify 3D interaction. A 3D representation of the mouse cursor is displayed at the tracked $3 \mathrm{D}$ position. A mouse click might be emulated if the position of the real finger and the visual representation of the finger stereoscopically displayed overlap in space. Alternatively, other gestures might be predefined, e.g., grab gestures. The depth information is also used when interacting with 2D GUIs. When using our framework, a corresponding depth is assigned to each window, and it is displayed stereoscopically. In addition shadows are added to all windows to further increase depth perception. When finger tracking is activated, the user can arrange windows on the desktop in depth by pushing or pulling them with a tracked finger. Figure 2 shows screenshots of two stereoscopic facetop interaction scenarios. Both users arrange windows on the desktop by pushing them with the finger.

\subsection{Combined Interaction Strategies}

By using the described concepts we are able to combine desktop devices with gestures. This setup is beneficial in scenarios where the user holds a virtual object in her non-dominant hand using universal exploration gestures (see Section 4.1), while the other hand can perform precise interactions via the mouse (see Figure 11). In contrast to using only common desktop devices, no context switches are required, e.g., to initiate status switches between navigation and manipulation modi. The roles of the hands may also change, i.e., the dominant hand can be used for gestures, whereas the non-dominant interacts via the keyboard.

Stereoscopic Mouse Cursor. When using the described setup we experienced some drawbacks. One shortcoming when interacting with stereoscopic representations using desktop-based interaction paradigms is the monoscopic appearance of the mouse cursor, which disturbs the stereoscopic perception. Therefore we provide two different strategies to display the mouse cursor. The first one exploits a stereoscopic mouse cursor which hovers over 3D objects. Thus the mouse cursor is always visible on top of the objects surface, and when moving the cursor over the surface of a three-dimensional object, the user gets an additional shape cue about the object. The alternative is to display the cursor always at 

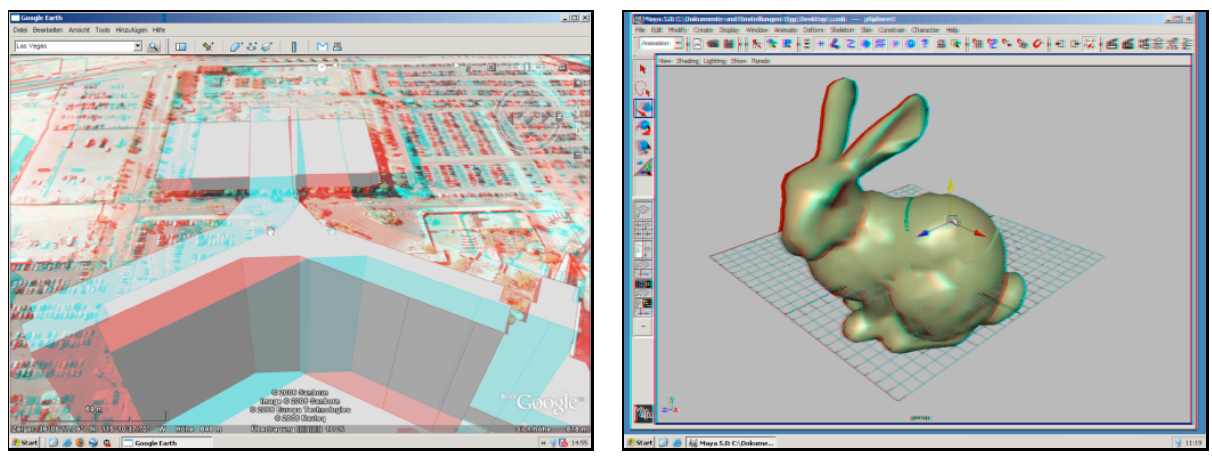

Fig. 3. Screenshots of the $3 \mathrm{D}$ user interface simultaneously showing monoscopic and stereoscopic content and (left) a stereoscopic mouse cursor and (right) a monoscopic interaction lens. To perceive a stereoscopic effect both images can be viewed with anaglyph glasses.

the image plane. In contrast to common desktop environments the mouse cursor gets invisible when it is obscured by another object extending out of the screen. Thus the stereoscopic impression is not disturbed by the mouse cursor, indeed the cursor is hidden during that time. Figure 3 (left) shows a stereoscopic scene in Google Earth where the mouse cursor is rendered stereoscopically on top of the building.

Monoscopic Interaction Lens. Many 2D as well as 3D applications provide interaction concepts which are best applicable in two dimensions using 2D interaction paradigms. One example are 3D widgets [7] which reduce simultaneously manipulated DoFs. Since these interaction concepts are optimized for 2D interaction devices and monoscopic viewing we propose a monoscopic interaction lens through which two-dimensional interactions can be performed without loosing the entire stereoscopic effect. We attach a lens to the position of the mouse cursor and project the content within such an arbitrarily shaped lens onto the image plane. Thus the user can focus on the given tasks and tools to perform $2 \mathrm{D}$ or 3D interactions in the same way as done on a common monoscopic display. This can be used to read text on a stereoscopic object, or to interact with $3 \mathrm{D}$ widgets.

\section{Implementation}

To provide a technical basis for the concepts described above, we explain some implementation details of our 3D user interface framework 19. To allow simultaneous viewing monoscopic content need to be modified in order to make it perceivable on AS displays, while a stereo pair needs to be generated from the $3 \mathrm{D}$ content. Since these are diverse image processing operations first 2D is separated from 3D content. To achieve this separation, our technique acts as an integrated layer between 3D application and OS. By using this layer we ensure 


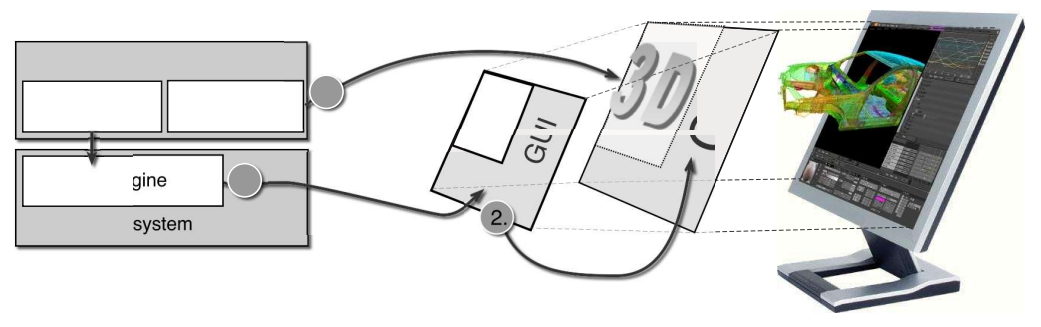

Fig. 4. Illustration of the interscopic user interface framework showing $2 \mathrm{D}$ and $3 \mathrm{D}$ content simultaneously

that the operating system takes care about rendering 2D GUI elements in a native way (see Figure 4 (step 1)).

Processing 2D Content. When viewing unadapted 2D content on AS displays the two images seen by the eyes are not correct half-images. This leads to an awkward viewing experience. To make this content perceivable we have to ensure that left and right eye see almost the same information, resulting in a flat two-dimensional image embedded in the image plane. To achieve this effect with (vertical-interlaced) AS displays the 2D content has to be scaled (see Figure 4 (step 2)) in order to ensure that the odd and even columns display almost the same information. With respect to the corresponding factor, scaling content can yield slightly different information for both half images. However, since differences in both images are marginal, the human vision system can merge the information to a final image which can be viewed comfortably. Since we achieve proper results for a resolution of $1024 \times 768$ pixels we choose this setting for a virtual desktop from which the content is scaled to the AS display's native resolution, i.e., $1600 \times 1200$ pixels. Therefore, we had to develop an appropriate display driver which allows the OS to mirror the desktop with the necessary resolution.

Generating Stereoscopic Images. Since only a few 3D applications natively support stereoscopic viewing on AS displays, in most cases we have to adapt also the $3 \mathrm{D}$ content in order to generate stereocopic images (see Figure 4 (step 3)). There are two techniques for making an existing 3D application stereoscopic. The first one is to trace and cache all 3D function calls and execute them twice, once for each eye. The alternative exploits image warping techniques. This technique performs a reprojection of the monoscopic image with respect to the values stored in the depth buffer. Image warping has the shortcoming that not all the scene content potentially visible from both eyes is presented in a single monoscopic image, and thus pixel filling approaches have to be applied [10. Hence, we use the first approach, catch all 3D function calls in a display list, apply off-axis stereographic rendering, and render the content in the even and odd columns for the left respectively right eye with respect to the head position as described in Section 4 . 

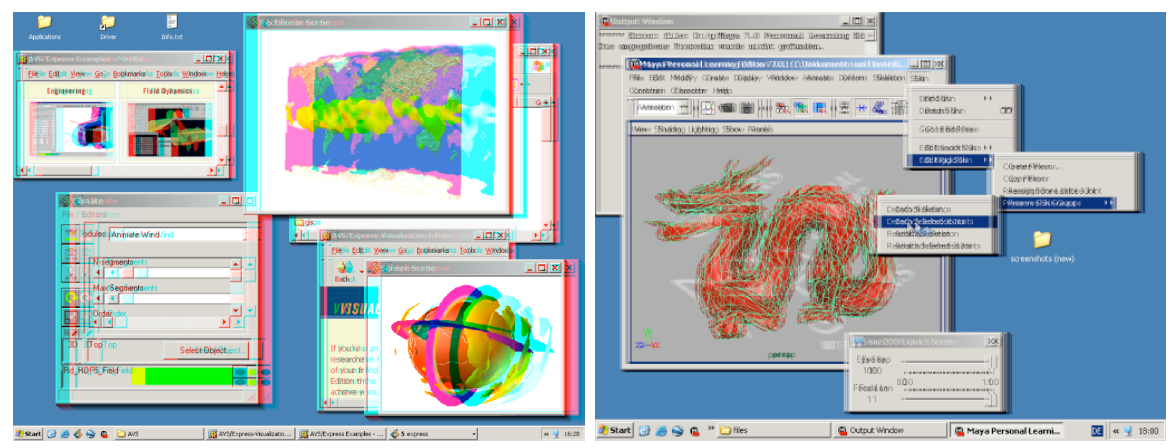

Fig. 5. Example screenshot of desktop with mono- as well as stereoscopic content shown (left) in anaglyph and (right) vertical interlaced mode

Embedding Mono- and Stereoscopic Display. To separate 2D and 3D content, we have to know which window areas are used for stereoscopic display. This can be determined either manually or automatically. When using the manual selection mechanism, the user is requested to add a 3D window or region and select it to be displayed stereoscopically with the mouse cursor. When using automatic detection, our framework seeks for 3D windows based on OpenGL and applies stereoscopic rendering.

The final embedding step of $2 \mathrm{D}$ and $3 \mathrm{D}$ content is depicted by step 3 in Figure 4. An obvious problem arises, when 2D and 3D content areas overlap each other. This may happen when either a pull-down menu or a context menu overlaps a 3D canvas. In this case the separation cannot only be based on the previous $3 \mathrm{D}$ window selection process. To properly render overlaying elements we apply a masking technique. This is for example important when dealing with 3D graphics applications using context menus to provide convenient access to important features. When merging $2 \mathrm{D}$ and $3 \mathrm{D}$ content the mask ensures that only those areas of the 3D window are used for stereoscopic display which are not occluded by 2D objects. Figure 5 shows two resulting screenshots in anaglyph respectively interlaced stereoscopic mode, where 3D content is shown in stereo. The windows appear at different distances to the user (see Section 4.2). The task bar and the desktop with its icons are rendered monoscopically.

\section{Preliminary Experiments}

In several informal tests all users have evaluated the usage of stereoscopic display for 3D applications as very helpful. In particular, two 3D modeling experts revealed stereoscopic visualization for 3D content in their 3D modeling environments, i.e., Maya and Cinema4D, as extremely beneficial. However, in order to evaluate the 3D user interface we have performed a preliminary usability study. We have used the described experimental environment (see Section 3). Furthermore, we have used a 3D mouse to enable precise 3D interaction. 

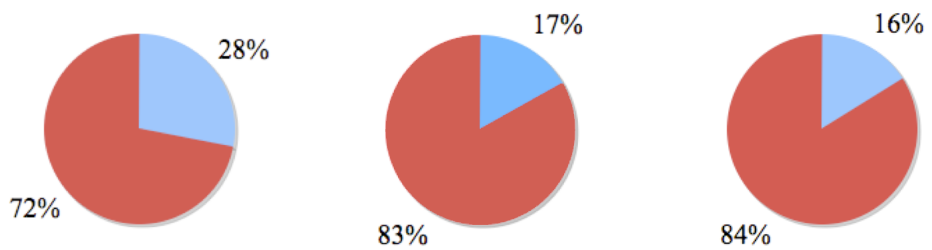

\section{a gestures $\square$ mouse/keyboard}

Fig. 6. Usage of gestures in comparison to traditional input devices constrained to (left) three DoFs, (middle) two DoFs and (right) one DoF

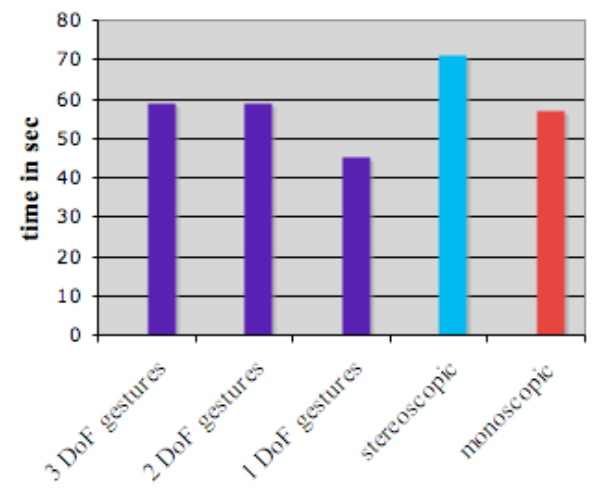

Fig. 7. Required time for the interaction task with stereoscopic display and gestures supporting three, two and one DoFs, and stereoscopic as well as monoscopic display only supporting mouse and keyboard without gesture

\subsection{Experimental Tasks}

We restricted the tasks to simple interactions in which four users had to delete several doors and windows from a virtual building. The building consisted of 290 triangles, where windows and doors (including 20 triangles) were uniformly separated. We have conducted three series. In the first series the user could use all provided input paradigms, i.e., mouse, keyboard, and gestures via a 3D mouse, in combination with stereoscopic visualization. In this series we have also performed subseries, where gestures were constrained to three, two and one DoFs. In the second series, only the mouse and keyboard could be used, again with stereoscopic display. In the last series, interaction was restricted to traditional devices with monoscopic visualization.

\subsection{Results}

We have measured the required time for the entire task and we have measured how long each input modality has been used. 
Figure 6 shows that as fewer DoFs are available fewer gestures have been used. When three DoFs were supported (left), one-third of the entire interaction time was spent on 3D manipulation by gestures with the objective to arrange the virtual building. With decreasing DoFs the required time for 3D manipulation also decreases. This is due to the fact that constraint-based interaction supports the user when arranging virtual objects. As pointed out in Figure 7 using gestures in combination with mouse and keyboard enhances performance, in particular when $3 \mathrm{D}$ manipulation is constrained appropriately. Participants accomplished the task fastest when all devices could be used and only one DoF was supported. Monoscopic display was advantageous in comparison to stereoscopic display. This does not come unexpected since exploration of 3D objects was hardly necessary; the focus was on simple manipulation where stereoscopic display was not essential.

\section{Discussion and Future Work}

In this paper we have introduced 3D user interface concepts which can be embedded in everyday working environments and provide an improved working experience. These strategies have the potential to be accepted by users as new user interface paradigms for specific tasks as well as for standard desktop interactions. The results of the preliminary evaluation indicate that the subjects are highly motivated to use the described framework, since as they remarked an instrumentation is not required. Moreover, users like the experience of using the 3D interface, especially the stereoscopic facetop approach. They evaluated the stereoscopic mouse cursor as clear improvement. The usage of the monoscopic interaction lens has been revealed as very useful because the subjects prefer to interact in a way which is familiar to them from working with common desktop systems.

In the future we will integrate further functionality and visual enhancements using more stereoscopic and physics-based motion effects. Moreover, we plan to examine further interaction techniques, in particular, for domain-specific interaction tasks.

\section{References}

1. Agarawala, A., Balakrishnan, R.: Keepin' It Real: Pushing the Desktop Metaphor with Physics, Piles and the Pen. In: Proceedings of the SIGCHI conference on Human Factors in computing systems, pp. 1283-1292 (2006)

2. Alpaslan, Z.Y., Sawchuk, A.A.: Three-Dimensional Interaction with Autostereoscopic Displays. In: Woods, A.J., Merritt, J.O., Benton, S.A., Bolas, M.R. (eds.) Proceedings of SPIE, Stereoscopic Displays and Virtual Reality Systems, vol. 5291, pp. 227-236 (2004)

3. Balakrishnan, R.: A Grant of 3D. Keynote speach Symposium on 3D User Interfaces (2006)

4. Benko, H., Ishak, E.W., Feiner, S.: Cross-Dimensional Gestural Interaction Techniques for Hybrid Immersive Environments. In: Proceedings of Virtual Reality, pp. 209-216. IEEE Computer Society Press, Los Alamitos (2005) 
5. Bourke, P.: Autostereoscopic Lenticular Images, http://local.wasp.uwa.edu.au/ pbourke

6. Bowman, D., Kruijff, E., LaViola, J., Poupyrev, I.: 3D User Interfaces: Theory and Practice. Addison-Wesley, Reading (2004)

7. Conner, D.B., Snibbe, S.C., Herndon, K.P., Robbins, D.C., Zeleznik, R.C., van Dam, A.: Three-Dimensional Widgets. In: Symposium on Interactive 3D Graphics (1992)

8. Dodgson, N.A.: Autostereoscopic 3D Displays. Computer 38(8), 31-36 (2005)

9. Hanson, A.J., Wernert, E.: Constrained 3D Navigation with 2D Controllers. In: Proceedings of Visualization '97, pp. 175-182. IEEE Computer Society Press, Los Alamitos (1997)

10. Kozankiewicz, P.: Fast Algorithm for Creating Image-based Stereo Images. In: Proceedings of WSCG, pp. 59-66 (2002)

11. Liu, J., Pastoor, S., Seifert, K., Hurtienne, J.: Three Dimensional PC toward novel Forms of Human-Computer Interaction. In: Three-Dimensional Video and Display Devices and Systems SPIE (2000)

12. Mulder, J.D., van Liere, R.: Enhancing Fish Tank VR. In: Proceedings of Virtual Reality, pp. 91-98. IEEE Computer Society Press, Los Alamitos (2000)

13. Peterka, T., Kooima, R.L., Girado, J.I., Ge, J., Sandin, D.J., Johnson, A., Leigh, J., Schulze, J., DeFanti, T.A.: Dynallax: Solid State Dynamic Parallax Barrier Autostereoscopic VR Display. In: Proceedings of the Virtual Reality, pp. 91-98. IEEE Computer Society Press, Los Alamitos (2007)

14. Philips 42-3D6C01, http://www.inition.co.uk

15. Project Looking Glass, http://www.sun.com/software

16. Robertson, G., van Dantzich, M., Robbins, D., Czerwinski, M., Hinckley, K., Risden, K., Thiel, D., Gorokhovsky, V.: The Task Gallery: A 3D Window Manager. In: Proceedings of SIGCHI Conference on Human Factors in Computing Systems, pp. 494-501 (2000)

17. Salzman, T., Stachniak, S., Stürzlinger, W.: Unconstrained vs. Constrained 3D Scene Manipulation. In: 8th IFIP International Conference on Engineering for Human-Computer Interaction, vol. 2254 (2001)

18. Smith, G., Salzman, T., Stürzlinger, W.: 3D Scene Manipulation with Constraints. In: Fisher, B., Dawson-Howe, K., O’Sullivan, C. (eds.) Virtual and Augmented Architecture, pp. 35-46 (2001)

19. Steinicke, F., Ropinski, T., Bruder, G., Hinrichs, K.: Interscopic User Interface Concepts for Fish Tank Virtual Reality Systems. In: Proceedings of the Virtual Reality, pp. 27-34 (2007)

20. Stotts, D., Smith, J.C., Gyllstrom, K.: FaceSpace: Endo- and Exo-Spatial Hypermedia. In: the Transparent Video Facetop Proceedings of the 15th ACM Conference on Hypertext and Hypermedia, pp. 48-57 (2004)

21. Szalavári, Z., Gervautz, M.: Using the Personal Interaction Panel for 3D Interaction. In: Proceedings of the Conference on Latest Results in Information Technology, p. 36 (1997)

22. Tramberend, H.: A Display Device Abstraction for Virtual Reality Applications. In: Proceedings of Afrigraph, pp. 75-80 (2001)

23. van Berkel, C.: Touchless Display Interaction. In: SID 02 DIGEST (2002) 